\title{
Antioxidant Formulae, Shengmai San, and LingGuiZhuGanTang, Prevent MPTP Induced Brain Dysfunction and Oxidative Damage in Mice
}

\author{
Vijayasree Vayalanellore Giridharan, ${ }^{1,2}$ \\ Rajarajan Amirthalingam Thandavarayan, ${ }^{2,3}$ and Tetsuya Konishi, ${ }^{2,4}$ \\ ${ }^{1}$ J.K.K. Nattraja College of Pharmacy, Komarapalayam, Tamil Nadu 638 183, India \\ ${ }^{2}$ Niigata University of Pharmacy \& Applied Life Sciences, Niigata 956 8603, Japan \\ ${ }^{3}$ Department of Cardiovascular Sciences, Houston Methodist Research Institute, Houston, TX 77030, USA \\ ${ }^{4}$ Changchun University of Chinese Medicine, Bosuo Road No. 1035, Jingyue Economic Development District, Changchun 130117, China \\ Correspondence should be addressed to Tetsuya Konishi; konishi@nupals.ac.jp
}

Received 11 June 2015; Revised 12 August 2015; Accepted 11 October 2015

Academic Editor: Pierre Champy

Copyright (c) 2015 Vijayasree Vayalanellore Giridharan et al. This is an open access article distributed under the Creative Commons Attribution License, which permits unrestricted use, distribution, and reproduction in any medium, provided the original work is properly cited.

\begin{abstract}
The present study was designed to evaluate the preventive effect of antioxidative traditional oriental medicine formulae, Shengmai San (SMS) and LingGuiZhuGanTang (LGZGT), against 1-methyl-4-phenyl-1,2,3,6-tetrahydropyridine (MPTP) (i.p $30 \mathrm{mg} \cdot \mathrm{kg}^{-1}$ for 5 consecutive days) induced neurotoxicity. In in vitro antioxidant assays measured with Trolox and butyl hydroxyl toluene as reference antioxidant revealed that SMS has higher scavenging potential against hydroxyl radical than superoxide anion radical, but LGZGT was the reverse. The neuroprotective effect of SMS and LGZGT against MPTP was evaluated in mice by behavioral, biochemical, and immunohistochemical studies. In the behavioral study, both SMS and LGZGT significantly reversed the locomotive impairment induced by MPTP. Simultaneously, both formulae significantly prevented the MPTP induced dopaminergic neuron loss assessed by tyrosine hydroxylase in the midbrain. Both SMS and LGZGT significantly attenuated the elevated lipid peroxidation and protein carbonyls levels by MPTP. The DNA damage induced by MPTP was also prevented by both formulae. Although a little difference in the protective functions was observed between the two formulae, such as in DNA damage and behavioral studies, the results indicate that both SMS and LGZGT with antioxidant property act as a good candidate applicable for the antioxidant based complementary therapies of neurodegenerative diseases.
\end{abstract}

\section{Introduction}

Parkinson's disease (PD) is a common adult-onset neurodegenerative disorder and over the period of time patients see their disability gradually growing and their quality of life declining [1]. The cause of idiopathic PD is still unknown, but complex features including aging, environmental factors, oxidative stress, neuroinflammation, and genetic factors are involved in the development of the disease [2,3]. Reactive oxygen species (ROS) and free radicals are implicated as the species playing critical roles in etiology and pathogenesis of age related neurodegenerative diseases, including PD [4-6].
The 1-methyl-4-phenyl-1,2,3,6-tetrahydropyridine (MPTP) administration in rodents is one of the models that mimics the PD phenotype. Numerous studies have shown that the administration of MPTP causes neuronal cell defect in the substantia nigra pars compacta (SNpc) $[4,7]$ and decline in locomotive activity and rearing frequencies $[8,9]$. ROS produced in the physiological metabolisms of MPTP is the major factor causing these pathological conditions; hence, overproduction of ROS causes oxidative stress to cellular critical molecules such as DNA, lipids, and proteins leading to many disorders [10]. Therefore, the antioxidant protection might be a primary strategy for treating or preventing PD. 
On the other side, current approaches to the treatment of PD suffer from the "wearing off" effect. A majority of patients treated with levodopa experience motor fluctuations, dyskinesia, or other complications after a period of time [11, 12]. So, it is mandatory to discover alternate pharmacological agents for the treatment of PD that will be effective either alone or in combination with presently used drugs to achieve maximum benefit with least side effects.

Traditional oriental medicines such as traditional Chinese medicines (TCM) and Kampo medicines are the composite formula of herbs and have been used historically for treating wide variety of disorders with an aim of enhancing physiological homeostatic potential. The brain is playing an important role in the physiological homeostasis and is also known as a weak organ against oxidative stress. Further, the pathogenic process of degenerative disorders is complex and involves many factors [1]. The antioxidant based composite therapy will thus be a promising approach against neurodegenerative diseases. Since different formulae are often used for the same disease condition and different pathological condition is treated by the same formula in the traditional oriental medicines, the oxidative stress is implicated as the common and basic target of them [13]. In this sense, the antioxidant traditional medicines are an attractive target of study as the model of antioxidant composite formula applicable for degenerative brain damage.

In the present study, we focused on the protective potential of two TCM formulae with different property against MPTP induced brain damages. Shengmai San (SMS), comprising three crude drug components, Radix Ginseng (Panax ginseng; Araliaceae), Radix Ophiopogonis (Ophiopogon japonicus; Liliaceae), and Fructus Schisandrae (Schisandra chinensis; Schisandraceae), has been traditionally used for treating heart disease $[14,15]$. LingGuiZhuGanTang (LGZGT), on the other hand, is composed of four crude drug components, Cinnamon twig (Cinnamomum cassia Presl.; Lauraceae), Atractylodis rhizoma (Atractylodes macrocephala Koidz.; Compositae), Glycyrrhizae radix (Glycyrrhiza uralensis Fisch.; Leguminosae), and one fungi, Hoelen (Poria $\operatorname{cocos}$ (Schw.) Wolf Polyporaceae) which is often used for diseases related to edema, chronic bronchitis, heart failure, and chronic nephritis [16]. The antioxidant and neuroprotective potentials of these formulae were studied in MPTP induced PD model mice using behavioral, biochemical, and immunohistochemical studies to assess the beneficial use of these antioxidant composite formulae in complimentary or integrated therapy of degenerated brain disorders.

\section{Materials and Methods}

2.1. Animals. Male C57BL/6 mice (40 animals; $12-15$ weeks old; 25-30 g) were purchased from SLC Inc., Japan. The animals were randomly housed in groups of five in polypropylene cages with wood shavings as bedding and maintained in a temperature controlled room $\left(22 \pm 2^{\circ} \mathrm{C}\right)$ on a $12 \mathrm{~h}$ light dark cycle (lights on at 7:00 am). The animals had free access to water and food throughout the experiment and were used after a 1 week adaptation period. The studies were carried out in accordance with the guidelines of the Committee on the Care and Use of Laboratory Animals, Niigata University of Pharmacy and Applied life Sciences, Japan.

2.2. Materials. SMS and LGZGT are commercially available ready to eat granules and were generously provided by Iskura Co., Ltd., Japan, and by Kotaro Co., Ltd., Japan, respectively. 5,5 Dimethyl 1 pyrroline $\mathrm{N}$ oxide (DMPO) was purchased from Labotec Co., Ltd., Japan. 6-Hydroxy-2,5,7,8 tetramethylchroman-2-carboxylic acid (Trolox) was purchased from Aldrich Chem. Co. Anti-dinitrophenyl (DNP) IgG developed in rabbit, and 2,4-dinitrophenylhydrazine (DNPH) and MPTP were from Sigma Co., Ltd., USA. Rabbit antimouse IgG conjugated to horseradish peroxidase (HRP) was obtained from Zymed, USA. 3,3',5,5' Tetramethylbenzidine (TMB) was obtained from Bio-Rad Lab., USA. All other chemicals were purchased from Wako Pure chemicals Industries Co., Ltd., Japan.

2.3. Experimental Protocol. In this study, the mice were randomly divided into $4(n=10)$ groups: group 1: normal control + vehicle (water treatment), group 2: MPTP treatment alone (30 $\mathrm{mg} \cdot \mathrm{kg}^{-1}$ i.p for 5 consecutive days), group 3: SMS + MPTP treatment, and group 4: LGZGT + MPTP treatment. The animals received the SMS and/or LGZGT treatment on day 1 to day 10. SMS and/or LGZGT granules were suspended in water and administered intragastrically at the dose of $8 \mathrm{~g} \cdot \mathrm{kg}^{-1}$ to the respective groups. The dose was selected from our previous studies $[17,18]$. The MPTP treatment was given to the same animals from day 6 to day 10 . On day 11, the animals were subjected to behavioral study at $24 \mathrm{~h}$ after last injection of MPTP and undergone biochemical assay $(n=5)$ and immunohistochemical study $(n=5)$.

\subsection{Hydroxyl Radical Scavenging Activity Measurement In} Vitro. Fenton reaction was used for the production of $\mathrm{HO}^{\bullet}$ and its scavenging activity was determined as reported previously [19]. DMPO used as a spin trap, in the X-Band Microwave Unit, JEOL JES-TE 200 ESR spectrometer for the detection of the electron spin resonance (ESR) spectra of DMPO-OH with the following settings: microwave power; $8 \mathrm{~mW}$, microwave frequency; $9.41 \mathrm{GHz}$, modulation amplitude; $0.1 \mathrm{mT}$, time constant; $0.03 \mathrm{~s}$, sweep time; $30 \mathrm{~s}$, center fields; 340.0/335.4 mT.

\subsection{Superoxide Scavenging Activity Measurement In Vitro.} Hypoxanthine and xanthine oxidase was used for the generation of superoxide and its scavenging activity was measured according to the methods reported previously [20]. DMPO was used as a spin trap. The ESR settings for DMPO$\mathrm{OOH}$ was microwave power; $8 \mathrm{~mW}$, microwave frequency; $9.41 \mathrm{GHz}$, modulation amplitude; $0.1 \mathrm{mT}$, time constant; 0.03 s, sweep time; 30 s, center fields; 340.0/335.4 mT.

2.6. DPPH Radical Scavenging Assay In Vitro. DPPH radical scavenging activity of SMS and LGZGT was determined according to the method of Shin et al. [21]. Briefly, $1 \mathrm{mg} \cdot \mathrm{mL}^{-1}$ 
of SMS and/or LGZGT was added to $3 \mathrm{~mL}$ of DPPH $(0.15 \mathrm{mM})$ solution. The mixtures were shaken vigorously and left to stand at room temperature in the dark for $30 \mathrm{~min}$. The change of DPPH concentration was monitored at $517 \mathrm{~nm}$ wavelength.

2.7. Open Field Test. The test was used to determine motorfunction alterations of animals. Open field test was done on a rectangular box $(40 \times 50 \times 63 \mathrm{~cm})$ and its floor was divided into $20(10 \times 10)$ small rectangles. The mice were placed in one of the corners of the rectangular box and the number of crossings between the small rectangles and the number of times the animals stood using their hind paws also called as rearing were determined. Further, the immobility time was measured by the lack of movement in seconds during testing and latency to start the movement was determined by the time taken for the animal to leave the first rectangle. All these parameters were determined by hand operated counters for the duration of $5 \mathrm{~min}$ [22]. The rectangular box was washed with a $5 \%$ alcohol solution before placing each animal.

2.8. Pole Test. The pole test for bradykinesia was conducted by using a modification of the reported method [23]. In a rough surfaced pole the mouse was placed upward $(10 \mathrm{~mm}$ diameter and $55 \mathrm{~cm}$ height), and $T_{\text {turn }}$ (time from the beginning of movement until the mouse turns completely downward) and $T_{\mathrm{LA}}$ (time until it arrives at floor) were measured. The elongation of these parameters is considered to reflect bradykinesia. This test was performed five times successively for each mouse [24].

2.9. Tissue Homogenate Preparation. The animals were sacrificed after behavioral study, and the whole brain was removed. The midbrain and cortex were dissected and then chilled in ice-cold saline. After washing with $0.85 \% \mathrm{NaCl}$, the tissue was suspended in cold $0.05 \mathrm{M}$ phosphate buffer containing $1.15 \%$ $(\mathrm{w} / \mathrm{v}) \mathrm{KCl}\left(9 \mathrm{~mL} \cdot \mathrm{g} \mathrm{g}^{-1}\right.$ wet tissue) and then homogenized at $4^{\circ} \mathrm{C}$ using an ULTRA TRAX homogenizer. The protein concentration of the homogenate was determined by BCA protein assay using bovine serum albumin as a standard.

2.10. Measurement of Lipid Peroxidation. The lipid peroxidation product in brain homogenates was assayed by measurement of the malondialdehyde (MDA) concentration according to the method elsewhere [25]. Brain homogenates $\left(0.4 \mathrm{mg}\right.$ of protein $\left.\cdot \mathrm{mL}^{-1}\right)$ were mixed with $1 \mathrm{~mL}$ of $1 \%$ thiobarbituric acid in $50 \mathrm{mM}$ sodium hydroxide and $1 \mathrm{~mL}$ of $2.8 \%$ trichloroacetic acid, and the concentration of MDA was measured. The MDA concentration is expressed as nanomoles per milligram of protein.

2.11. Measurement of Protein Carbonyl Content. A sensitive enzyme linked immunosorbent assay (ELISA) was employed to determine protein carbonyl content as previously reported [26]. Briefly, the supernatants of tissue homogenate obtained after centrifugation were incubated with $1 \%$ streptomycin sulfate. The homogenate (1 $\mathrm{mg}$ as protein) was suspended in PBS (phosphate buffer) and allowed to react with $10 \mathrm{mM}$
$\mathrm{DNPH}$ in $2 \mathrm{~N} \mathrm{HCl}$ in darkness at room temperature for $1 \mathrm{~h}$. The protein was precipitated with $20 \%$ trichloro acetic acid and then solubilized in PBST (PBS + tween 20) and was placed in 96-well plate and incubated overnight at $4^{\circ} \mathrm{C}$. The samples were incubated $4 \mathrm{~h}$ with a primary antibody (anti-DNP rabbit $\operatorname{IgG}$ ) at $37^{\circ} \mathrm{C}$ and washed with PBST, then reacted with a secondary antibody (anti-mouse rabbit IgG HRP conjugate) for $1 \mathrm{~h}$. Peroxidase reactions were performed with the addition of TMB for an hour and stopped with the addition of sulphuric acid. Absorbance was measured at $450 \mathrm{~nm}$ using a Bio-Rad model 550 microplate reader.

2.12. Alkaline Single-Cell Gel Electrophoresis Assay. DNA damage was detected by alkaline single-cell gel electrophoresis assay (Comet assay) [27, 28]. Briefly, brain tissue was removed immediately after sacrificing the mice and washed with chilled PBS. The tissue was dissociated into cells using a cell dissociation sieve-tissue grinder kit under ice-cold conditions and processed for the Comet assay. Approximately, $1 \times$ $10^{5}$ cells were mixed with $1.5 \mathrm{~mL}$ of $0.8 \%$ low melting agarose solution prepared in $0.9 \%$ saline at $38^{\circ} \mathrm{C}$ and poured onto fully frosted microscope slides. After solidification, slides were immersed in lysis buffer $(2.5 \mathrm{M} \mathrm{NaCl}, 100 \mathrm{mM} \mathrm{Na} 2$-EDTA, $1 \%$ Triton X-100, and $10 \%$ dimethyl sulfoxide) for $1 \mathrm{~h}$ at $4^{\circ} \mathrm{C}$ and electrophoresed in alkaline buffer $(300 \mathrm{mM} \mathrm{NaOH}, 1 \mathrm{mM}$ $\mathrm{Na}_{2}$-EDTA, pH 13) using $25 \mathrm{~V}, 400 \mathrm{~mA}$ for $30 \mathrm{~min}$. After electrophoresis, the slides were washed gently in a neutralizing buffer (0.4 M Tris-HCl, $\mathrm{pH}$ 7.5) to remove the alkaline buffer and detergent and stored on wet tissue paper in a closed plastic box at $4^{\circ} \mathrm{C}$ until observation. The slides were stained with SYBR Green II, and at least 50 cells were captured per slide at $\times 200$ magnification using a fluorescence microscope (Olympus (BH2-RFCA), Japan). The digital imaging CASP software (http://casp.sourceforge.net/) was used to measure the indexes of DNA damage. Tail length and tail moment were selected as the parameters to quantify DNA damage.

2.13. Tyrosine Hydroxylase (TH) Immunohistochemistry. After the behavioral study, the mice were anesthetized with ketamine and were intracardially perfused with saline and the entire mesencephalon was removed and fixed using $4 \%$ paraformaldehyde. The paraffin sections ( $16 \mu \mathrm{m}$ thick) encompassing were prepared for $\mathrm{TH}$ immunohistochemical staining. Following blocking with $8 \%$ skimmed milk for $40 \mathrm{~min}$ at room temperature, sections were incubated with rabbit anti-TH antibody (diluted $1: 200$ ) at $4^{\circ} \mathrm{C}$ overnight. The avidin-biotin immunoperoxidase method was used for the detection of positive reaction with the Vectastain ABC Kit (Vector Laboratories, Burlingame, CA) according to manufacturer's instructions. The bound peroxidase was stained with $0.05 \%, 3.3^{\prime}$-diaminobenzidine with $0.03 \%$ hydrogen peroxide in Tris-HCl buffer, $\mathrm{pH}$ 7.6, followed by counterstaining with methyl green [29]. The number of $\mathrm{TH}$-positive neurons in 4 sections of the $\mathrm{SN}$ in each mouse was counted under light microscopy at a magnification of $\times 400$. The average of right and left neuronal cells (4 sections) was regarded as the positive cells number of each animal. The mean number of TH-positive neurons for each 
TABLE 1: In vitro antioxidant activities of SMS and LGZGT.

\begin{tabular}{lcccc}
\hline Antioxidant assays & SMS IC $_{50}(\mu \mathrm{g} / \mathrm{mL})$ & LGZGT IC $_{50}(\mu \mathrm{g} / \mathrm{mL})$ & Trolox IC $_{50}(\mu \mathrm{g} / \mathrm{mL})$ & $\mathrm{BHT} \mathrm{IC}_{50}(\mu \mathrm{g} / \mathrm{mL})$ \\
\hline Hydroxyl radical scavenging activity & 45.6 & 98.6 & 18.9 & 34.3 \\
Superoxide scavenging activity & 298.6 & 103.6 & 100.0 & 98.4 \\
DPPH scavenging activity & 34.7 & 15.8 & 10.8 & 20.0 \\
\hline
\end{tabular}

The antioxidant activities were determined as described in Section 2.

representative mesencephalic section was calculated three times under the final magnification of $\times 400$.

2.14. Statistical Analysis. All the results were expressed as mean \pm standard error (SE). The data were analyzed using one-way ANOVA followed by Tukey's post hoc test and Student's (unpaired) $t$-test. $P<0.05$ was considered as statistically significant.

\section{Results}

3.1. In Vitro Antioxidant Potential of TCM Formulae. The formation of superoxide (DMPO-OOH) and hydroxyl radical (DMPO-OH) spin adducts were inhibited by the addition of SMS and/or LGZGT and the $\mathrm{IC}_{50}$ was evaluated. The results showed that both SMS and LGZGT showed comparable antioxidant potential with Trolox and butyl hydroxyl toluene (BHT) measured as reference antioxidant, but both formulae showed slight different specificity towards radical species. LGZGT showed rather stronger superoxide radical scavenging activity and SMS, on the other hand, showed potent scavenging activity against hydroxyl radicals. DPPH radical scavenging potential was almost the same for both formulae. The $\mathrm{IC}_{50}$ values of SMS, LGZGT and reference antioxidants are summarized in Table 1.

3.2. Effect of TCM Formulae on Neurobehavioral Study. The preventive effect of SMS and/or LGZGT on MPTP induced locomotive dysfunction and dyskinesia was studied by open field test and pole test, respectively. As was observed elsewhere $[8,9]$, the MPTP group exhibited a significant decrease in locomotion, rearing frequencies $(P<0.001$, Figures 1 (a) and $1(b))$ as compared to control group in the open field test. However, both SMS + MPTP and LGZGT + MPTP groups showed a significant recovery in the locomotion and rearing frequencies $(P<0.05)$ compared to the MPTP group. In addition, SMS $(P<0.001)$ and LGZGT $(P<0.001)$ groups showed a significant decrease in immobility time and latency to start the movement when compared to the MPTP group (Figures 1(c) and 1(d)).

The protective effect of SMS and LGZGT against MPTP induced dyskinesia was examined by the pole test and the results shown in Figure 2. The MPTP group exhibited increase in the time taken in both $T_{\text {turn }}(P<0.01)$ and $T_{\mathrm{LA}}$ $(P<0.05)$ as compared to control group. Treatment with SMS significantly $(P<0.05)$ reduced the time taken both in $T_{\mathrm{LA}}$ and $T_{\text {turn }}$ as compared to MPTP treated group (Figures 2(a) and 2(b)). LGZGT also reduced $T_{\mathrm{LA}}$ and $T_{\text {turn }}$, but only the effect was significant in $T_{\text {turn }}$ (Figures 2(a) and 2(b)).
3.3. Effect of TCM Formulae on Oxidative Stress in Brain. Oxidative damage production in the brain of MPTP treated mice was assessed by measuring MDA and protein carbonyls formation as lipid peroxidation and protein oxidation markers, respectively. On the day 11, 5 animals from each group were undergone biochemical estimation after behavioral study. The mice treated with MPTP markedly increased the MDA $(P<0.001)$ and protein carbonyls $(P<0.05)$ levels in the midbrain (Figures 3(a) and 3(b)). However, a little regional diversity was observed between MDA and protein carbonyl formation in the brain such that in midbrain; both MDA and carbonyl formations were significantly increased, but, in cortex, only carbonyls formation was increased as in the midbrain although the increase in cortex was not statistically significant. Treatment with SMS and/or LGZGT suppressed both MDA $(P<0.001)$ and carbonyls formations $(P<0.05)$ to the levels comparable to these of control mice.

3.4. Protective Effect of TCM on DNA Damages Assed by Comet Assay. DNA damage was further assessed for the brain cells using Comet assay as another marker of brain oxidative damage caused by MPTP. The length of Comet tail is proportional to the extent of DNA strand breaks. The DNA damage measured in terms of tail length $(P<0.01)$, and tail moment $(P<0.05)$ was significantly increased in the mice treated with MPTP as compared to control. The MPTP induced DNA damage was significantly decreased in both SMS $(P<0.01)$ and LGZGT $(P<0.01)$ treated groups in terms of tail length (Figure 4(f)).

3.5. Preventive Effect of TCM on MPTP Induced Dopaminergic Neuron Loss in SNpc. The effects of SMS and LGZGT on the number of $\mathrm{TH}$-immunoreactive dopaminergic neurons were examined immunohistochemically and the results are summarized in Figure 5, in order to rationale the preventive effect of TCM on the above observed behavioral dysfunction. On the day 11, 5 animals from each group have undergone immunohistochemical study after behavioral test. There was a significant $(P<0.01)$ loss of TH-immunoreactive neurons in the SNpc of MPTP treated mice, as compared to control (Figures 5(a) and 5(b)). The treatment with SMS $(P<0.05)$ and/or LGZGT significantly $(P<0.05)$ prevented the loss of dopaminergic neurons and the cell densities were comparable to those of control group (Figures 5(c) and 5(d)).

\section{Discussion}

In the present study, we examined the protective effect of two TCM formulae, SMS and LGZGT against MPTP induced neurotoxicity. Results from behavioral, biochemical, 


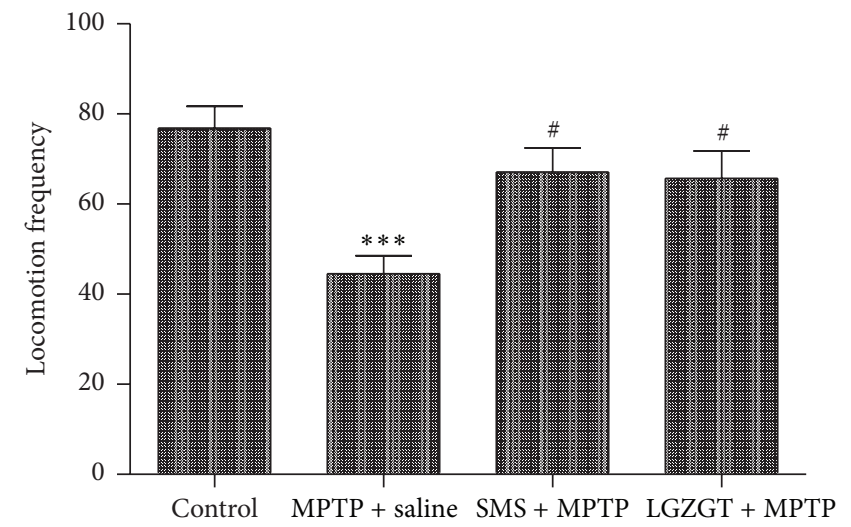

(a)

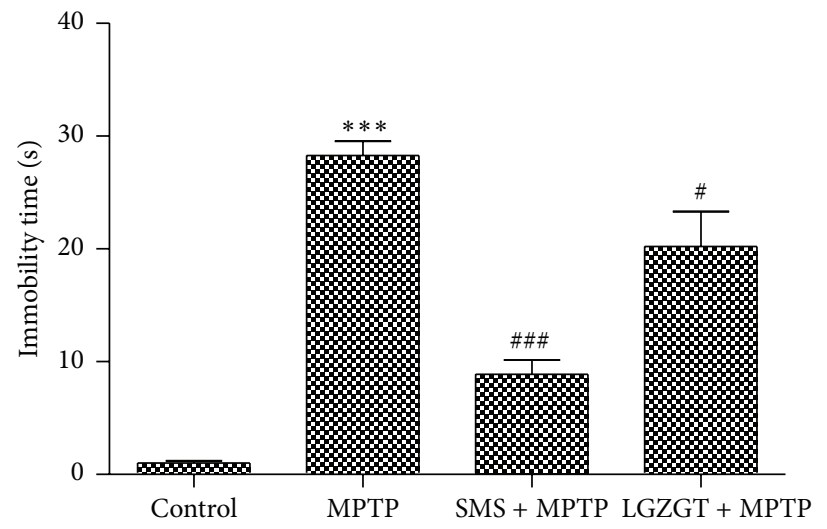

(c)

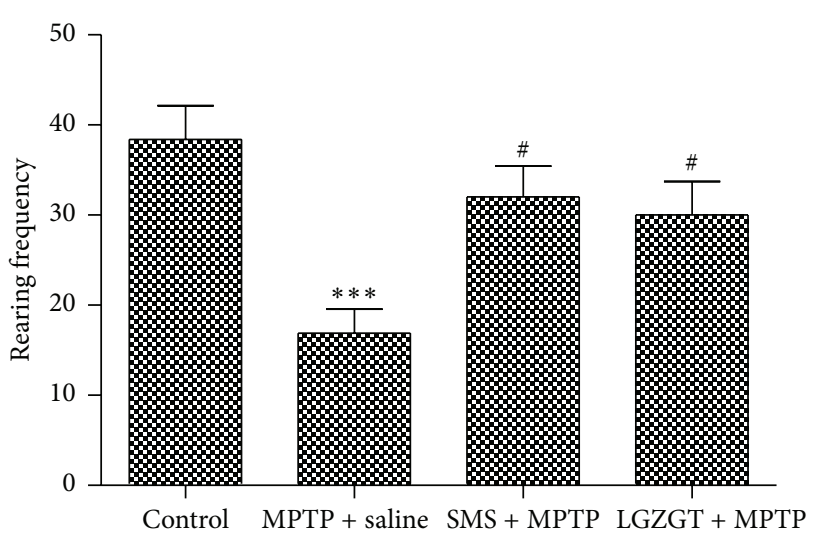

(b)

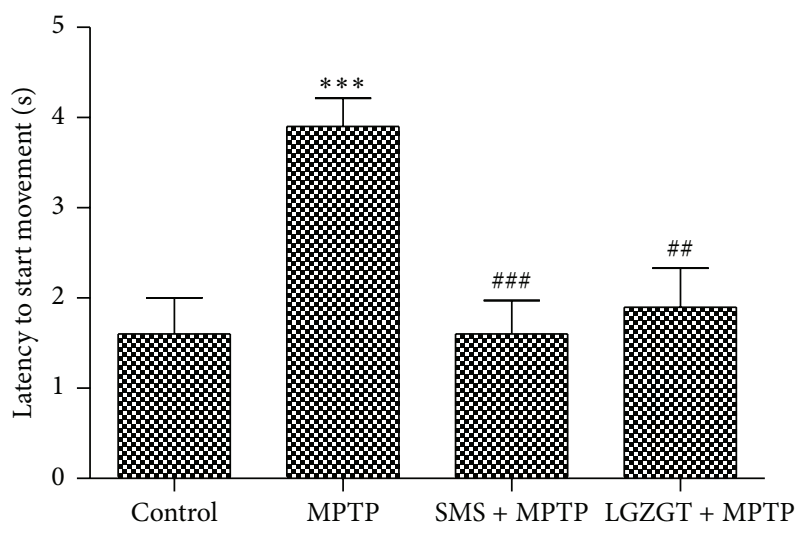

(d)

FIGURE 1: Effects of SMS and LGZGT on general motor activity impairment in MPTP treated mice (open field test): (a) locomotion frequency; (b) rearing frequency; (c) immobility time; (d) latency to start moment. Each value represents the mean \pm SE. $N=10$, ${ }^{* * *} P<0.001$ : significantly different from control group. ${ }^{\# \#} P<0.001,{ }^{\# \#} P<0.01$, and ${ }^{\#} P<0.05$ : significantly different from the MPTP only treated group.

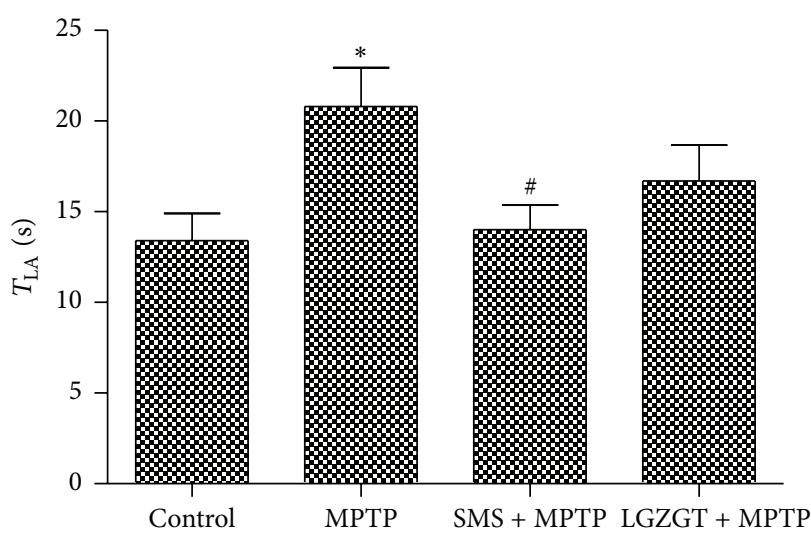

(a)

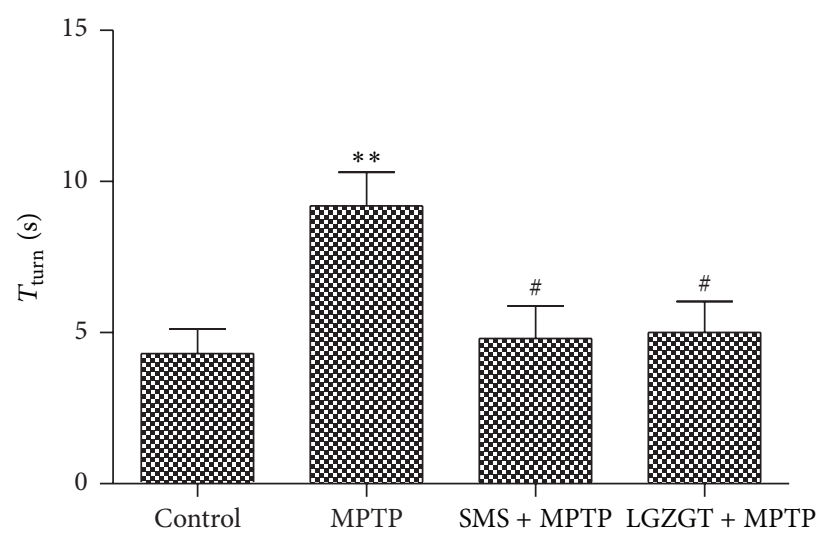

(b)

FIGURE 2: Effects of SMS and LGZGT on MPTP induced dyskinesia assessed by pole test. The mouse was placed head upward near the top of a rough surfaced pole. The time needed for the animal to turn completely downward $\left(T_{\text {turn }}\right)\left(\right.$ a) and to reach the floor $\left(T_{\mathrm{LA}}\right)(\mathrm{b})$ was determined. Each value represents the mean \pm SE. $N=10,{ }^{* *} P<0.01,{ }^{*} P<0.05$ : significantly different from control group. ${ }^{\sharp} P<0.05$ : significantly different from the MPTP only treated group. 


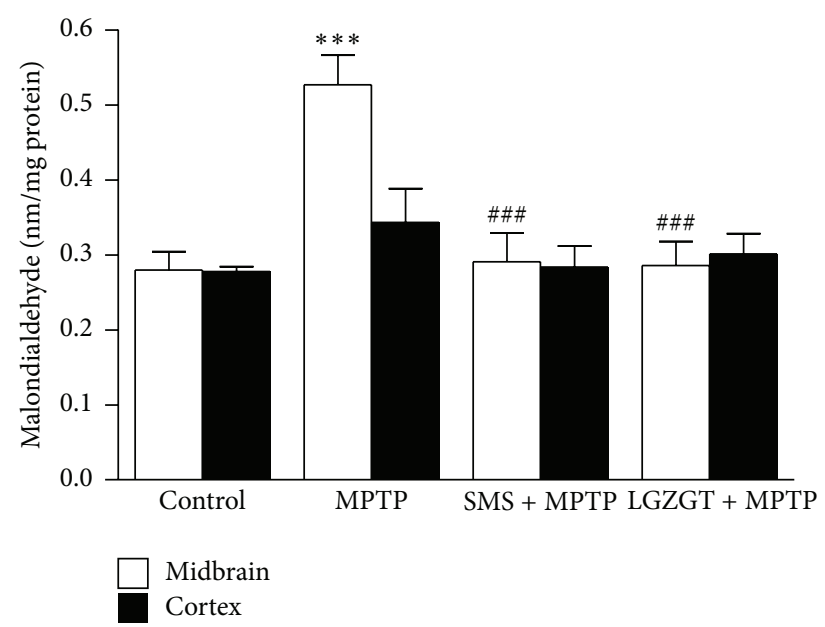

(a)

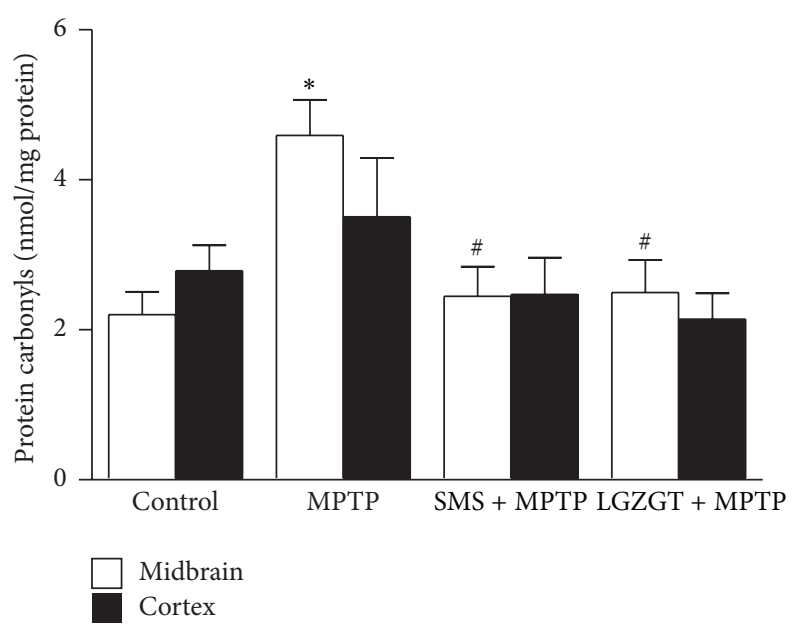

(b)

FIGURE 3: Prevention of MPTP induced oxidative stress in brain by SMS and LGZGT. Lipid peroxidation (a) and protein carbonyls (b) in tissue homogenates of midbrain and cortex were quantified as described in Section 2. Data represent nmolmg ${ }^{-1}$ protein and mean \pm SE. $N=5,{ }^{* * *} P<0.001,{ }^{*} P<0.05$, significantly differ from control group. ${ }^{\# \#} P<0.001,{ }^{\#} P<0.05$, significantly differs from the MPTP only treated group.

and immunohistochemical studies revealed that both SMS and/or LGZGT treatment were effective in attenuating MPTP induced neurotoxicity.

Extensive evidence suggest that protoxin MPTP is oxidized to 1-methyl-4-phenyl-2, 3-dihydropyridinium $\left(\mathrm{MDPD}^{+}\right)$ by monoamine oxidase $\mathrm{B}$ (MAO-B) after systemic administration. It is then converted to $\mathrm{MPP}^{+}$which has a high affinity substrate for dopamine transporter and for norepinephrine and serotonin transporters $[29,30] . \mathrm{MPP}^{+}$also induces mitochondrial dysfunction with a pathologic cascade involving both excitotoxicity and free radical production. It was reported that the brains of mice treated with MPTP exhibited the decreased complex I activity, the increased hydroxyl radical production, and the elevated activities of superoxide dismutase (SOD), catalase, and glutathione peroxidase (GPx) $[31,32]$.

Indeed, there are reports that the levels of markers of oxidative damage to proteins, such as carbonyl modifications of soluble proteins, are significantly increased in postmortem samples of SNpc in PD [33,34]. Therefore, the oxidative stress is accepted as the critical factor in pathogenesis of PD, and several antioxidants have been examined to protect against MPTP induced neurotoxicity including natural antioxidant like curcumin $[3,35,36]$.

However, PD is a multifactorial disease involving oxidative/nitrative stress, mitochondrial dysfunction, protein aggregation, proteasome inhibition, and so forth. Most of these pathways are interlinked, but the molecular details of their relationships are still remained unclear $[1,4]$. Moreover, neither the contribution nor the chronologies of all the pathways in neurodegeneration have been ascertained yet. Therefore, researchers have been screening for drugs that are therapeutically effective against most of these pathways. This emphasized the necessity of agents that are simultaneously effective against multiple pathways in PD [36].

In this sense, TCM prescriptions with antioxidant potential are attractive target of study as an alternative treating modality of cerebral degenerative diseases such as PD and Alzheimer's disease. They are basically antioxidant based multiple functional formula because they are comprised of several herbal constituents with different functional property, and many of herbs are usually antioxidant active that will be essential function for protecting neuronal cell against oxidative stress.

The TCM formulae, SMS, and LGZGT studied in the present experiments are used for different disease conditions. LGZGT has been frequently applied for the symptoms associated with brain functions such as dizziness and SMS clinically for the treatment of coronary heart diseases [1416]. Moreover, both formulae had strong antioxidant activity as the $\mathrm{IC}_{50}$ values were comparable to reference antioxidants, Trolox, and BHT in vitro, but their scavenging properties were somewhat different. SMS was rather specific scavenger of hydroxyl radical, but LGZGT showed stronger activity towards superoxide and DPPH radicals in vitro (Table 1).

The neuroprotective function of SMS has been reported elsewhere, such that it protects rat brain against heart stroke-induced arterial hypotension and cerebral ischemia by inhibiting the prooxidant enzyme nitric oxide synthase [37]. In our earlier studies, SMS was shown to upregulate the antioxidant enzymes such as glutathione reductase, GPx, and SOD against scopolamine induced amnesia [18]. In the present study, not only SMS but also LGZGT with differential radical scavenging property prevented oxidative tissue damages in the brain (midbrain and cortex) of mice treated with MPTP as shown by the inhibitory action on formation of 


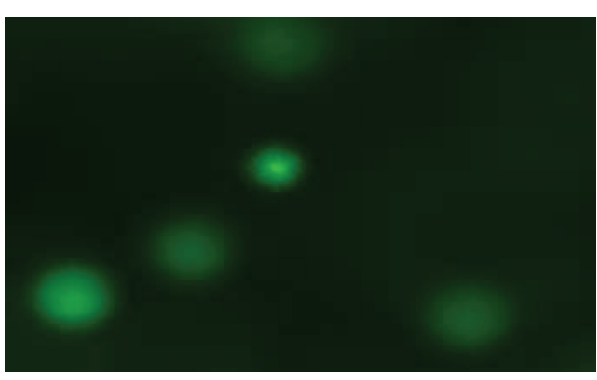

(a)

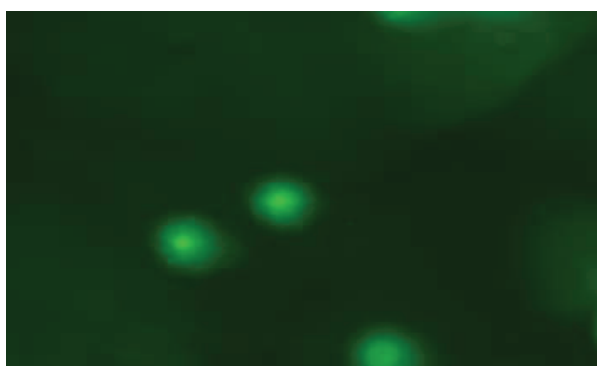

(c)

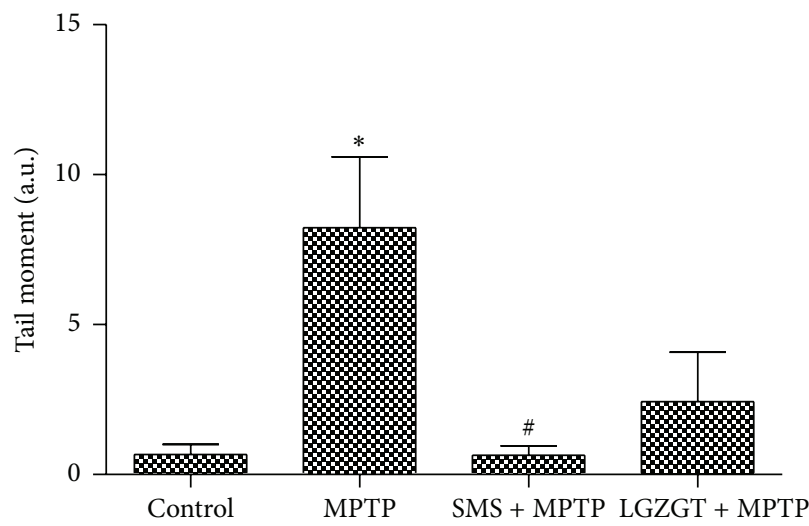

(e)

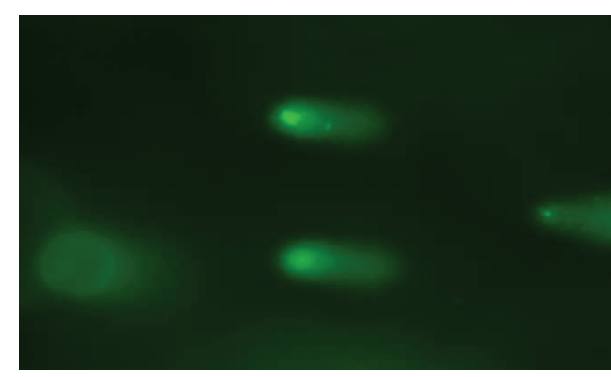

(b)

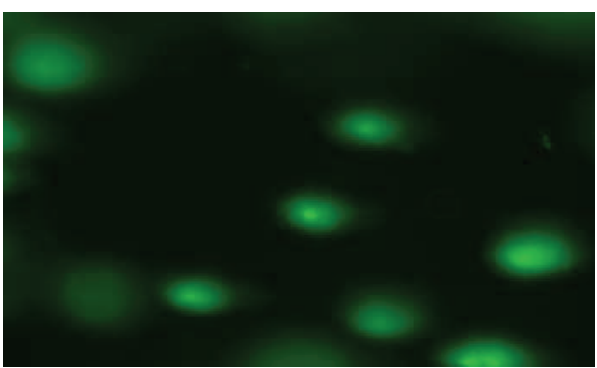

(d)

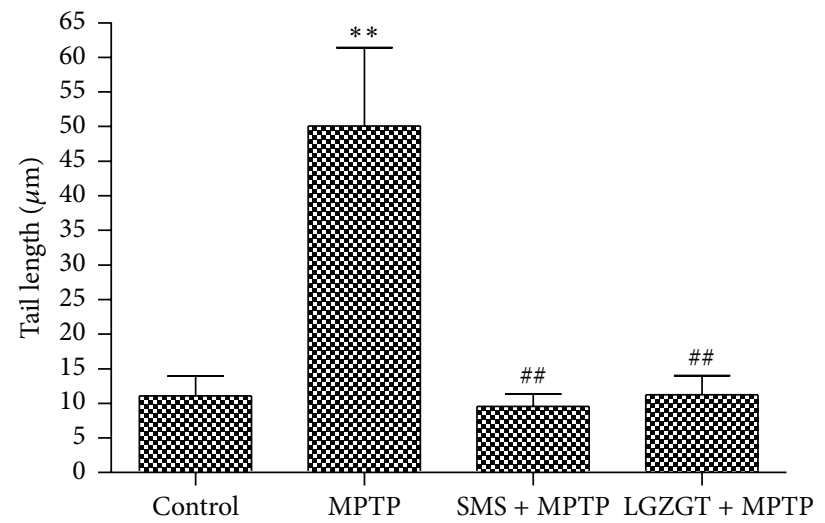

(f)

FIGURE 4: Prevention of MPTP induced DNA damage by SMS and LGZGT using Comet assay. DNA damage was determined by alkaline single-cell gel electrophoresis assay (Comet assay) in brain tissue, (a) control, (b) MPTP + saline, (c) SMS + MPTP, and (d) LGZGT + MPTP. Typical figures are at 200x original magnification. (e) Tail moment, (f) tail length. Values are mean \pm SE. $N=4,{ }^{* *} P<0.01,{ }^{*} P<0.05$ : significantly different from control group. ${ }^{\# \#} P<0.01,{ }^{\#} P<0.05$ : significantly different from the MPTP only treated group.

MDA and protein carbonyls (Figure 3). Further study on the DNA damage protection also indicated effective antioxidant property of both TCM formulae in vivo (Figure 4).

These antioxidant properties were finely reflected in their protective action on MPTP induced neurobehavioral impairments in MPTP model mice of PD used elsewhere [38, 39] as it is predicted from the previous reports that MPTP induced neurotoxicity associated with oxidative stress and DNA damage [40, 41]. MPTP treatment significantly decreased the locomotion and rearing frequencies and increased the latency to start the first movement and immobility time (Figures 1 and 2), but both TCM formulae significantly prevented the behavioral impairments induced by MPTP. Along with this observation, the MPTP induced loss of dopaminergic neurons in the $\mathrm{SNpc}$ was also prevented by
SMS and LGZGT (Figure 5). Age related MPTP neurotoxicity has been hypothesized to be due to an increase of $\mathrm{MPP}^{+}$ concentration with age, because young mice excluded $\mathrm{MPP}^{+}$ rapidly than older ones [42]. In the present study, mice of age about $12-15$ weeks were selected. The percentage reduction of $\mathrm{TH}$ neurons was $40 \%$ as compared to control; this is consistent with $\mathrm{He}$ et al. [42]. Further, there was not much observed difference in their protective effect on MPTP induced oxidative damages but for a slight difference in behavioral results between both formulae. These results suggest the multifunctional property of antioxidant formulae will be another factor in their protective function.

Currently, antioxidant compounds are widely recognized for the potential therapeutic treatment of diseases associated with oxidative stress. Several, antioxidant drugs, natural 


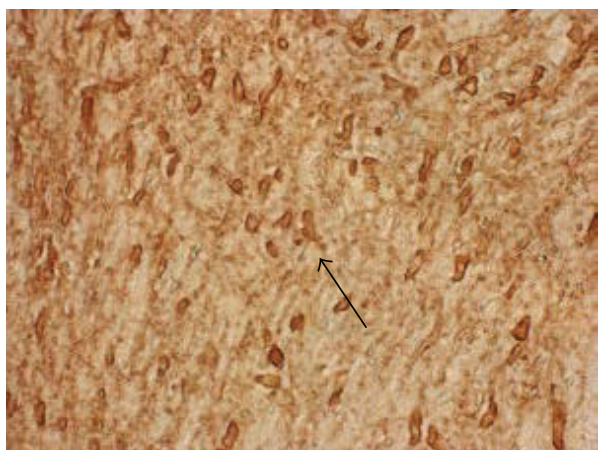

(a)

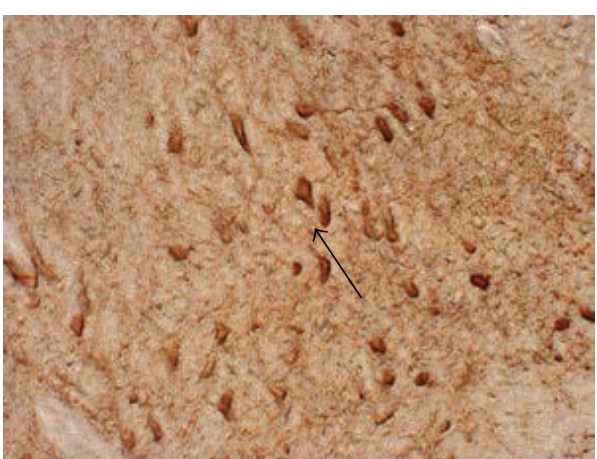

(c)

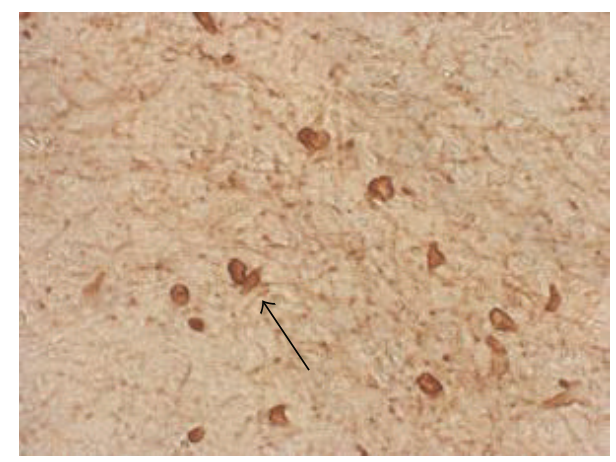

(b)

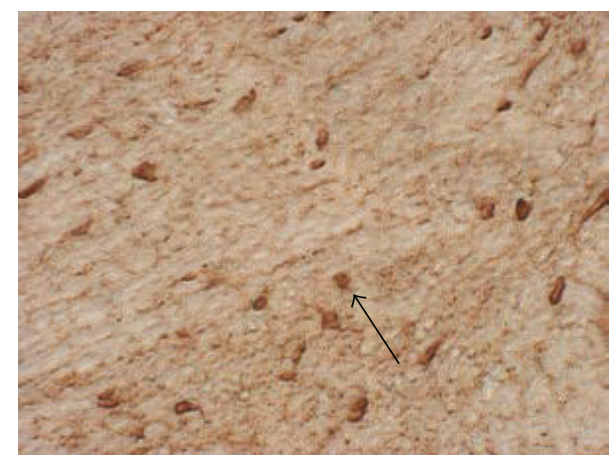

(d)

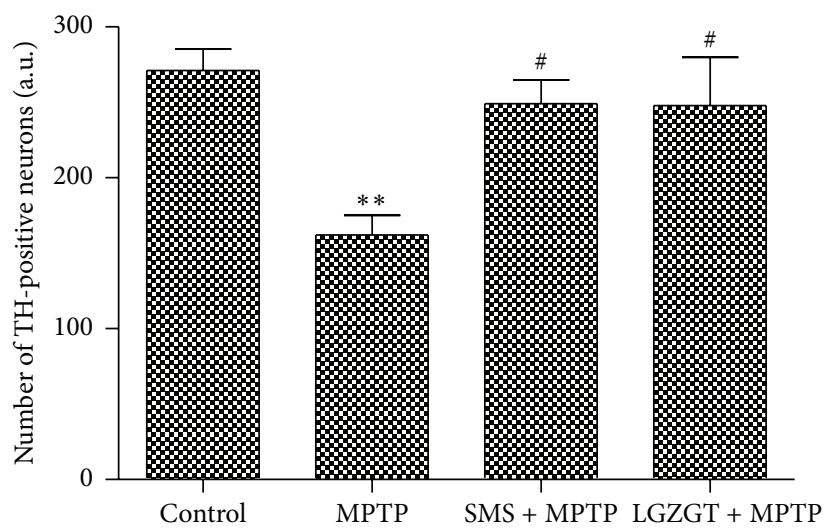

(e)

FIGURE 5: Preventive effect of SMS and LGZGT on MPTP induced dopaminergic neurons loss in SNpc. Dopaminergic neurons were immunohistochemically determined by the antibody reactive to tyrosine hydroxylase as described in the method section. (a) Control, (b) MPTP + saline, (c) SMS + MPTP, and (d) LGZGT + MPTP. Values are mean \pm SE. $N=5,{ }^{* *} P<0.01$ : significantly different from control group. ${ }^{\#} P<0.05$ : significantly different from the MPTP only treated group.

products, and antioxidant materials in foods have been tested in the MPTP mouse model $[43,44]$. Sch B, a lignan isolated from Schisandra chinensis that is one of the herbal components of SMS, was also active in protecting oxidative damage and functional deficit of mice brain induced by cisplatin [45]. In contrast, TCM prescriptions are usually prescribed with multiple herbal components. Therefore, complex interactions, such as additive, synergistic, and antagonistic interactions are involved in the activities of the component herbs. These interactions are considered essential in improving their therapeutic potentiality and in reducing the side effects of certain toxic ingredients [46]. It is not yet understandable whether the mixtures are more advantageous than a single molecule. Therefore, further studies are required to clarify the underlying molecular mechanism of components interaction and develop an appropriate system to allow the evaluation of the preventive and/or ameliorative potential of such mixed formulae against complex diseases including PD.

\section{Conclusion}

The present study revealed that two traditional oriental formulae, Shengmai San and LingGuiZhuGanTang, covering different pathological applications, one is used for brain 
related disorder and the other is used for heart related disorder, with differential radical reactivity, effectively prevented MPTP induced cerebral oxidative stress and neurobehavioral impairments. Although further studies are required, the present observations suggest the potential use of these antioxidant oriental medicines to treat degenerative diseases in complementary and integrated medicine.

\section{Conflict of Interests}

The authors declare that they have no conflict of interests.

\section{Acknowledgments}

This study was supported by a grant to Tetsuya Konishi from the Promotion and Mutual Aid Corporation for Private Schools. The authors thank the Rotary Yoneyama Scholarship Association for the financial assistance to Vijayasree Vayalanellore Giridharan. They acknowledge Ms. Xu Bing and Dr. Haruyo Ichikawa at NUPALS for their contributions to the experiments and discussion.

\section{References}

[1] B. Zhao, "Natural antioxidants protect neurons in Alzheimer's disease and Parkinson's disease," Neurochemical Research, vol. 34, no. 4, pp. 630-638, 2009.

[2] W. Dauer and S. Przedborski, "Parkinson's disease: mechanisms and models," Neuron, vol. 39, no. 6, pp. 889-909, 2003.

[3] H.-M. Gao, B. Liu, W. Zhang, and J.-S. Hong, "Novel antiinflammatory therapy for Parkinson's disease," Trends in Pharmacological Sciences, vol. 24, no. 8, pp. 395-401, 2003.

[4] J. D. Adams Jr., M. L. Chang, and L. Klaidman, "Parkinson's disease-redox mechanisms," Current Medicinal Chemistry, vol. 8, no. 7, pp. 809-814, 2001.

[5] M. R. Cookson and P. J. Shaw, "Oxidative stress and motor neurone disease," Brain Pathology, vol. 9, no. 1, pp. 165-186, 1999.

[6] M. Ebadi, S. K. Srinivasan, and M. D. Baxi, "Oxidative stress and antioxidant therapy in Parkinson's disease," Progress in Neurobiology, vol. 48, no. 1, pp. 1-19, 1996.

[7] R. Heikkila, A. Hess, and R. Duvoisin, "Dopaminergic neurotoxicity of 1-methyl-4-phenyl-1,2,5,6-tetrahydropyridine in mice," Science, vol. 224, no. 4656, pp. 1451-1453, 1984.

[8] D. C. German, E. L. Nelson, C.-L. Liang, S. G. Speciale, C. M. Sinton, and P. K. Sonsalla, "The neurotoxin MPTP causes degeneration of specific nucleus A8, A9 and A10 dopaminergic neurons in the mouse," Neurodegeneration, vol. 5, no. 4, pp. 299312, 1996.

[9] M. S. Gevaerd, E. Miyoshi, R. Silveira, N. S. Canteras, R. N. Takahashi, and C. Da Cunha, "L-Dopa restores striatal dopamine level but fails to reverse MPTP-induced memory deficits in rats," The International Journal of Neuropsychopharmacology, vol. 4, no. 4, pp. 361-370, 2001.

[10] K. Cui, X. Luo, K. Xu, and M. R. Ven Murthy, "Role of oxidative stress in neurodegeneration: recent developments in assay methods for oxidative stress and nutraceutical antioxidants," Progress in Neuro-Psychopharmacology and Biological Psychiatry, vol. 28, no. 5, pp. 771-799, 2004.
[11] J. Jankovic, "Motor fluctuations and dyskinesias in Parkinson's disease: clinical manifestations," Movement Disorders, vol. 20, supplement 11, pp. S11-S16, 2005.

[12] J. Jankovic and L. G. Aguilar, "Current approaches to the treatment of Parkinson's disease," Neuropsychiatric Disease and Treatment, vol. 4, no. 4, pp. 743-757, 2008.

[13] T. Konishi, "Brain oxidative stress as basic target of antioxidant traditional oriental medicines," Neurochemical Research, vol. 34, no. 4, pp. 711-716, 2009.

[14] H. Ichikawa and T. Konishi, "In vitro antioxidant potentials of traditional Chinese medicine, Shengmai San and their relation to in vivo protective effect on cerebral oxidative damage in rats," Biological \& Pharmaceutical Bulletin, vol. 25, no. 7, pp. 898-903, 2002.

[15] Q. Ni, J. Wang, E.-Q. Li et al., "Study on the protective effect of shengmai san (see text) on the myocardium in the type 2 diabetic cardiomyopathy model rat," Journal of Traditional Chinese Medicine, vol. 31, no. 3, pp. 209-219, 2011.

[16] Z. H. Song, S. J. Dai, H. Q. Li, K. S. Bi, and D. Feng, "Study on the compatibility of composite herbal medicines of the lingguizhugan decoction," Zhongguo Zhong Yao Za Zhi, vol. 27, no. 10, pp. 760-762, 2002.

[17] L. Wang, G. Muxin, H. Nishida, C. Shirakawa, S. Sato, and T. Konishi, "Psychological stress-induced oxidative stress as a model of sub-healthy condition and the effect of TCM," Evidence-Based Complementary and Alternative Medicine, vol. 4, no. 2, pp. 195-202, 2007.

[18] V. V. Giridharan, R. A. Thandavarayan, and T. Konishi, "Effect of Shengmai-san on cognitive performance and cerebral oxidative damage in BALB/c mice," Journal of Medicinal Food, vol. 14, no. 6, pp. 601-609, 2011.

[19] M. A. Ali and T. Konishi, "Enhancement of hydroxyl radical generation in the fenton reaction by alpha-hydroxy acid," Biochemistry and Molecular Biology International, vol. 46, no. 1, pp. 137-145, 1998.

[20] E. Finkelstein, G. M. Rosen, and E. J. Rauckman, "Spin trapping of superoxide and hydroxyl radical: practical aspects," Archives of Biochemistry and Biophysics, vol. 200, no. 1, pp. 1-16, 1980.

[21] S. W. Shin, A. K. Ghimeray, and C. H. Park, "Investigation of total phenolic, total flavonoid, antioxidant and allyl isothiocyanate content in the different organs of Wasabi japonica grown in an organic system," African Journal of Traditional, Complementary and Alternative Medicines, vol. 11, no. 3, pp. 3845, 2014.

[22] M. Sedelis, R. K. Schwarting, and J. P. Huston, "Behavioral phenotyping of the MPTP mouse model of Parkinson's disease," Behavioural Brain Research, vol. 125, no. 1-2, pp. 109-125, 2001.

[23] N. Ogawa, Y. Hirose, S. Ohara, T. Ono, and Y. Watanabe, "A simple quantitative bradykinesia test in MPTP-treated mice," Research Communications in Chemical Pathology and Pharmacology, vol. 50, no. 3, pp. 435-441, 1985.

[24] K. Okuda, Y. Kotake, and S. Ohta, "Parkinsonism-preventing activity of 1-methyl-1,2,3,4-tetrahydroisoquinoline derivatives in C57BL mouse in vivo," Biological \& Pharmaceutical Bulletin, vol. 29, no. 7, pp. 1401-1403, 2006.

[25] V. V. Giridharan, R. A. Thandavarayan, S. Sato, K. M. Ko, and T. Konishi, "Prevention of scopolamine-induced memory deficits by schisandrin B, an antioxidant lignan from Schisandra chinensis in mice," Free Radical Research, vol. 45, no. 8, pp. 950958, 2011

[26] H. Buss, T. P. Chan, K. B. Sluis, N. M. Domigan, and C. C. Winterbourn, "Protein carbonyl measurement by a sensitive 
ELISA method," Free Radical Biology and Medicine, vol. 23, no. 3, pp. 361-366, 1997.

[27] R. C. Chaubey, H. N. Bhilwade, R. Rajagopalan, and S. V. Bannur, "Gamma ray induced DNA damage in human and mouse leucocytes measured by SCGE-Pro: a software developed for automated image analysis and data processing for Comet assay," Mutation Research, vol. 490, no. 2, pp. 187-197, 2001.

[28] R. A. Thandavarayan, K. Watanabe, F. R. Sari et al., "Modulation of doxorubicin-induced cardiac dysfunction in dominantnegative p38alpha mitogen-activated protein kinase mice," Free Radical Biology and Medicine, vol. 49, no. 9, pp. 1422-1431, 2010.

[29] V. Jackson-Lewis and S. Przedborski, "Protocol for the MPTP mouse model of Parkinson's disease," Nature Protocols, vol. 2, no. 1, pp. 141-151, 2007.

[30] D. S. Bredt and S. H. Snyder, "Isolation of nitric oxide synthetase, a calmodulin-requiring enzyme," Proceedings of the National Academy of Sciences of the United States of America, vol. 87, no. 2, pp. 682-685, 1990.

[31] D. S. Cassarino, C. P. Fall, R. H. Swerdlow et al., "Elevated reactive oxygen species and antioxidant enzyme activities in animal and cellular models of Parkinson's disease," Biochimica et Biophysica Acta-Molecular Basis of Disease, vol. 1362, no. 1, pp. 77-86, 1997.

[32] H. C. Hung and E. H. Lee, "MPTP produces differential oxidative stress and antioxidative responses in the nigrostriatal and mesolimbic dopaminergic pathways," Free Radical Biology \& Medicine, vol. 24, no. 1, pp. 76-84, 1998.

[33] Z. I. Alam, S. E. Daniel, A. J. Lees, D. C. Marsden, P. Jenner, and B. Halliwell, "A generalised increase in protein carbonyls in the brain in Parkinson's but not incidental Lewy body disease," Journal of Neurochemistry, vol. 69, no. 3, pp. 1326-1329, 1997.

[34] E. Floor and M. G. Wetzel, "Increased protein oxidation in human substantia nigra pars compacta in comparison with basal ganglia and prefrontal cortex measured with an improved dinitrophenylhydrazine assay," Journal of Neurochemistry, vol. 70, no. 1, pp. 268-275, 1998.

[35] B. Jagatha, R. B. Mythri, S. Vali, and M. M. S. Bharath, "Curcumin treatment alleviates the effects of glutathione depletion in vitro and in vivo: therapeutic implications for Parkinson's disease explained via in silico studies," Free Radical Biology and Medicine, vol. 44, no. 5, pp. 907-917, 2008.

[36] O. Vajragupta, P. Boonchoong, H. Watanabe, M. Tohda, N. Kummasud, and Y. Sumanont, "Manganese complexes of curcumin and its derivatives: evaluation for the radical scavenging ability and neuroprotective activity," Free Radical Biology and Medicine, vol. 35, no. 12, pp. 1632-1644, 2003.

[37] N. L. Wang, C. K. Chang, Y. L. Liou, C. L. Lin, and M. T. Lin, "Shengmai San, a Chinese herbal medicine protects against rat heat stroke by reducing inflammatory cytokines and nitric oxide formation," Journal of Pharmacological Sciences, vol. 98, no. 1, pp. 1-7, 2005.

[38] G. Feng, Z. Zhang, Q. Bao et al., "Protective effect of chinonin in MPTP-induced C57BL/6 mouse model of Parkinson's disease," Biological and Pharmaceutical Bulletin, vol. 37, no. 8, pp. 13011307, 2014.

[39] S. Nagarajan, D. R. Chellappan, P. Chinnaswamy, and S. Thulasingam, "Ferulic acid pretreatment mitigates MPTPinduced motor impairment and histopathological alterations in C57BL/6 mice," Pharmaceutical Biology, vol. 53, no. 11, pp. 15911601, 2015.
[40] L. Migliore, R. Scarpato, F. Coppede, L. Petrozzi, U. Bonuccelli, and V. Rodilla, "Chromosome and oxidative damage biomarkers in lymphocytes of Parkinson's disease patients," International Journal of Hygiene and Environmental Health, vol. 204, no. 1, pp. 61-66, 2001.

[41] L. Petrozzi, C. Lucetti, G. Gambaccini et al., "Cytogenetic analysis oxidative damage in lymphocytes of Parkinson's disease patients," Neurological Sciences, vol. 22, no. 1, pp. 83-84, 2001.

[42] X. J. He, H. Nakayama, M. Ueno, and K. Doi, "Age-dependent susceptibility to MPTP neurotoxicity in C57BL mice: a tyrosine hydroxylase-immunohistochemical evaluation," Journal of Toxicologic Pathology, vol. 17, no. 4, pp. 239-244, 2004.

[43] K. T. Lu, M. C. Ko, B. Y. Chen et al., "Neuroprotective effects of resveratrol on MPTP-induced neuron loss mediated by free radical scavenging," Journal of Agricultural and Food Chemistry, vol. 56, no. 16, pp. 6910-6913, 2008.

[44] S.-J. Tsai, W.-W. Kuo, W.-H. Liu, and M.-C. Yin, "Antioxidative and anti-inflammatory protection from carnosine in the striatum of MPTP-treated mice," Journal of Agricultural and Food Chemistry, vol. 58, no. 21, pp. 11510-11516, 2010.

[45] V. V. Giridharan, R. A. Thandavarayan, H. N. Bhilwade, K. M. Ko, K. Watanabe, and T. Konishi, "Schisandrin B, attenuates cisplatin-induced oxidative stress, genotoxicity and neurotoxicity through modulating NF-kappaB pathway in mice," Free Radical Research, vol. 46, no. 1, pp. 50-60, 2012.

[46] J.-T. Cheng, "Review: drug therapy in Chinese traditional medicine," The Journal of Clinical Pharmacology, vol. 40, no. 5, pp. 445-450, 2000. 


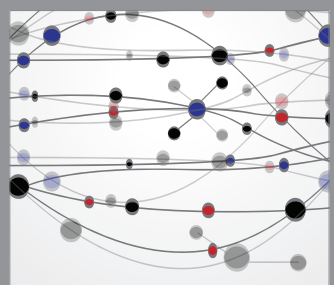

The Scientific World Journal
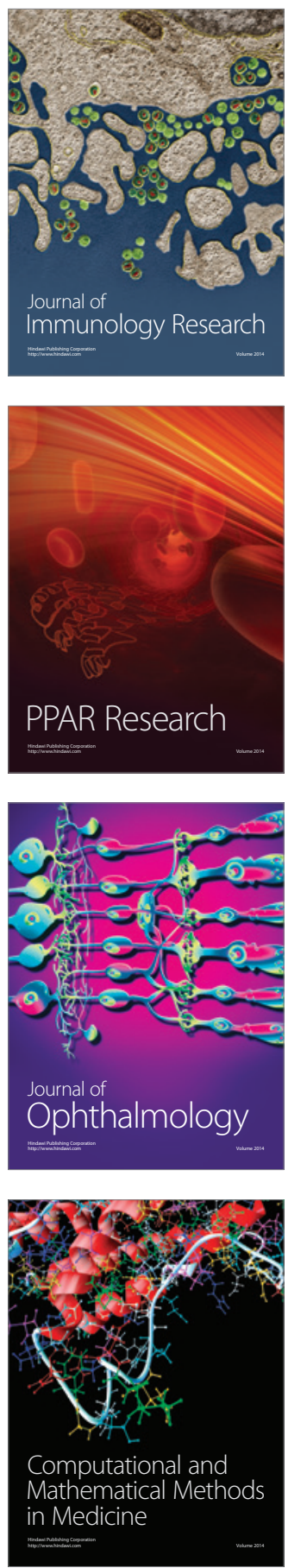

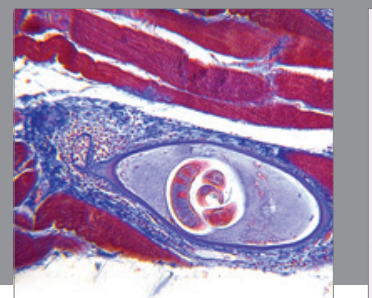

Gastroenterology

Research and Practice
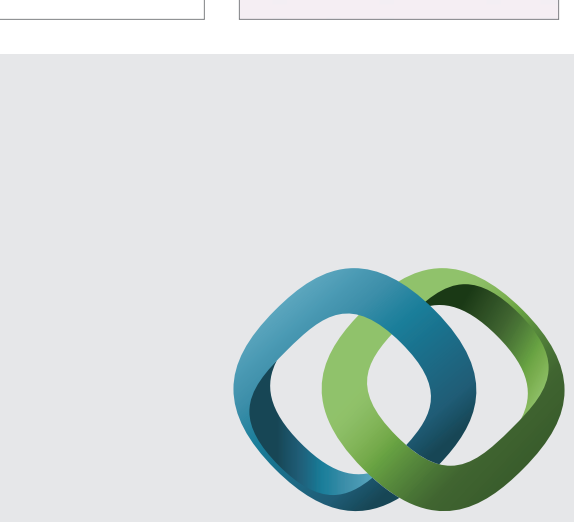

\section{Hindawi}

Submit your manuscripts at

http://www.hindawi.com
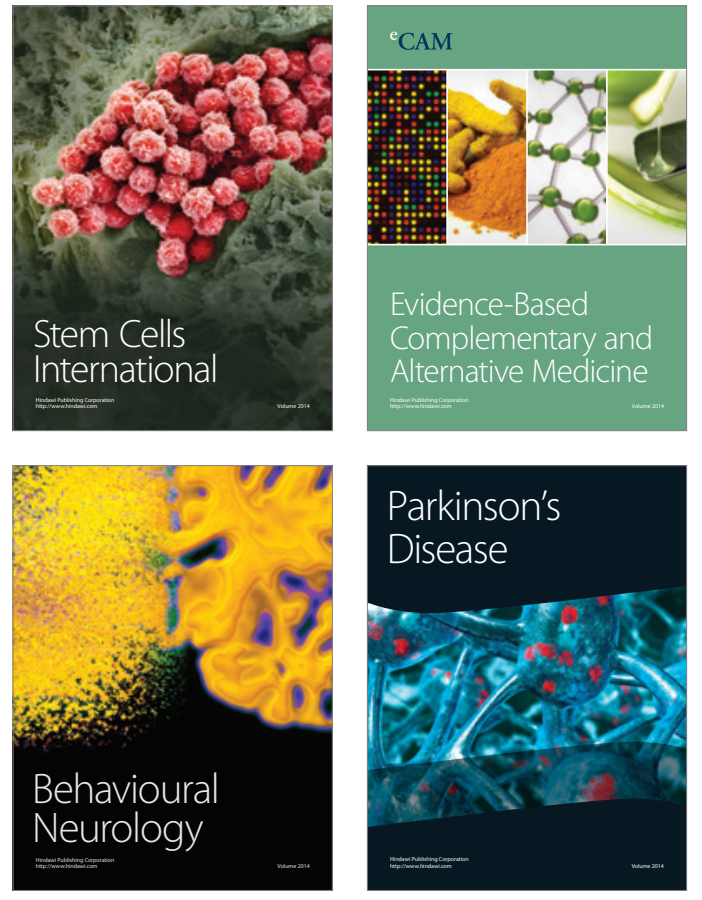
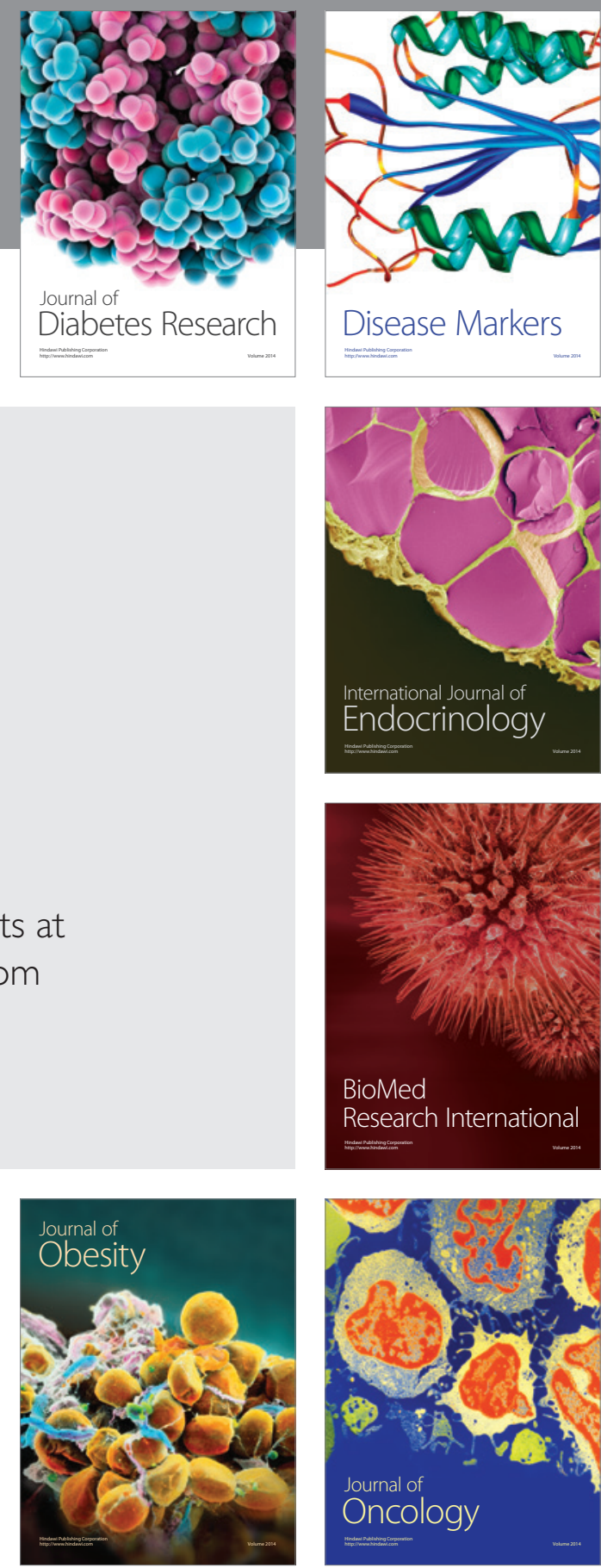

Disease Markers
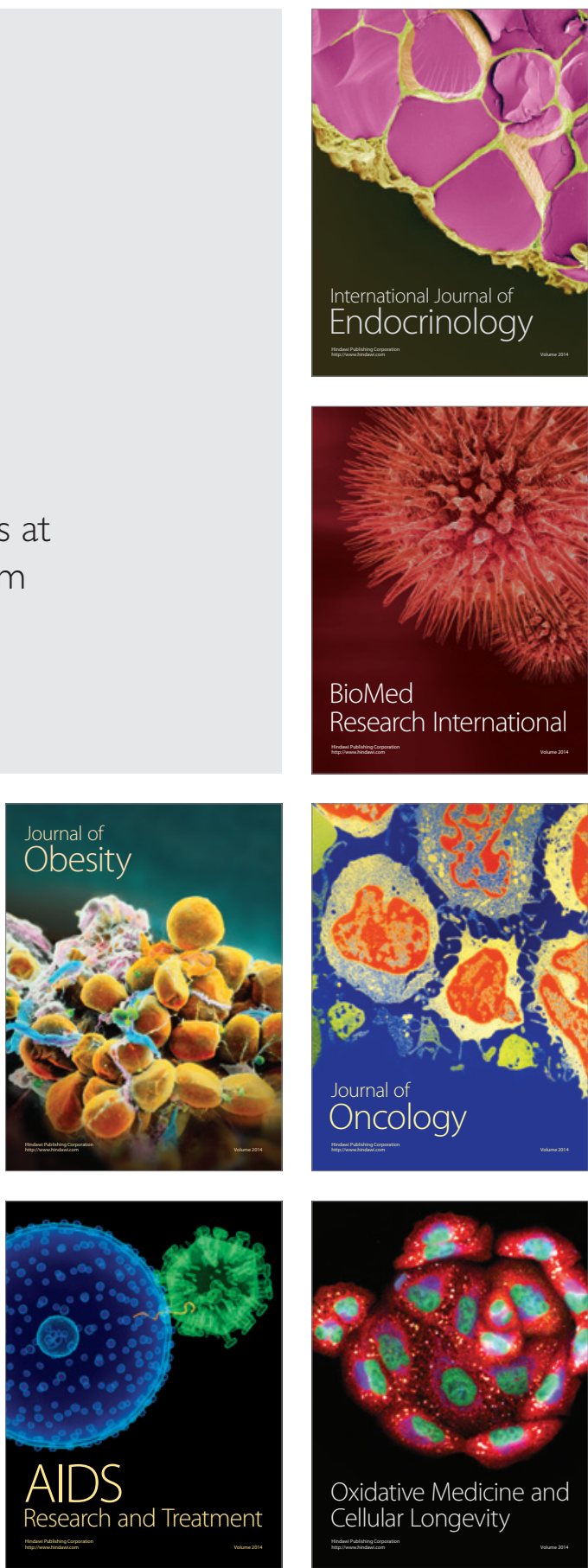